\title{
Wolman Disease
}

National Cancer Institute

\section{Source}

National Cancer Institute. Wolman Disease. NCI Thesaurus. Code C61271.

A very rare, autosomal recessive inherited lysosomal storage disease caused by mutations in the LIPA gene. Signs and symptoms appear in infancy and include developmental delay, vomiting, abdominal distention, hepatosplenomegaly, jaundice, anemia, steatorrhea, and calcification of the adrenal glands. 